\title{
Infrared Reflectivity of Pedestrian Mannequin for Autonomous Emergency Braking Testing
}

\author{
Terence Haran, Senior Member IEEE and Stanley Chien, Senior Member IEEE
}

\begin{abstract}
In order to be able to evaluate the performances of different Autonomous Emergency Braking (AEB) systems for pedestrian crash avoidance and mitigation, a standard surrogate pedestrian mannequin needs to be developed. One of the requirements for pedestrian mannequin is to ensure it "looks" like a real representative pedestrian to each of the sensor modalities used in AEB systems. The purpose of this paper is to generate the recommended IR reflectance specifications for the standard surrogate pedestrian mannequin based on the collected data from various sources and the experiments.
\end{abstract}

\section{INTRODUCTION}

Autonomous Emergency Braking (AEB) system will be standard or optional equipment on all vehicles in the near future. In order to evaluate and compare the performances of different AEB systems, standard test scenarios, methods and tools need to be developed. One essential testing tool needed is the surrogate pedestrian mannequin. There have been many ongoing efforts in developing the surrogate pedestrian mannequins [1-3] and several organizations (NHTSA, SAE, ISO, etc.) are creating mannequin standards. One of the requirements for pedestrian mannequin is to ensure it "looks" like a real representative pedestrian to LiDAR. However, to the best knowledge of the authors, there is not a published study that describes an infrared reflectivity specification to ensure that a pedestrian mannequin "looks" like a representative pedestrian to a LIDAR. The purpose of this paper is to derive the recommended IR reflectance specification for surrogate pedestrian mannequin based on the collected data from various sources and the experiments.

The paper is organized as follows: sections II specifies the problem in detail and provides background information. Section III describes the process for deriving the recommended IR reflectance of a pedestrian mannequin. Section IV describes the suggested measurement method for verifying IR reflectance. Section V concludes the paper. ${ }^{1}$

Terence Haran is with the Electro-Optical Systems laboratory, Georgia Tech Research Institute, Atlanta, GA 30332 USA. E-mail: terence.haran@gtri.gatech.edu.

Stanley Chien is with the Transportation Active Safety Institute, Indiana University - Purdue University Indianapolis, Indiana, USA. Email:schien@iupui.edu.

\section{BACKGROUND INFORMATION}

The goal of this paper is to specify the IR ${ }^{2}$ reflectance of the pedestrian mannequin for testing the performance of pedestrian detection of automotive LIDAR. We first describe the common types of LIDAR used in automotive applications and then describe the LIDAR operation principles. It should be clarified that infrared wavelengths discussed here are in the near infrared portion of the spectrum rather than the far infrared wavelengths typically used for body heat detection.

\section{A. Automotive LIDAR on the market}

Optical sensor technology is finding increasing utility for applications involving navigation and obstacle avoidance. These sensors generally fall into two categories: passive sensors, which include typical visible band cameras, that collect light either from reflection of ambient light from objects in a scene or that is generated by thermal emissions from the objects themselves; and active sensors, which use their own light sources to "probe" a scene in order to determine information about its contents. Light detector and ranging (equivalently, laser detection and ranging), or LIDAR, sensors are a type of active optical sensor in which laser beams are used to interrogate a scene to determine the location and shape of objects within it. This is typically performed by illuminating some (or all) of the scene with a laser pulse of short duration (typically on the order of nanoseconds) and then precisely measuring the time required for the laser signal to be reflected back to its source, and in some cases the intensity of the reflected signal. These measurements allow the determination of how far an object is from the instrument (based on the time of flight for the reflected laser pulse) as well as how reflective the target is (based on the intensity of the received signal). LIDAR sensors are similar in concept to radar systems but utilize optical frequencies instead of radio frequency sources. There are numerous LIDAR system designs in use today which vary widely based on their intended applications, but most used for automotive applications obtain data over a two dimensional scene either by scanning a small laser beam rapidly across the scene and measuring the reflected signal with a single detector (thus obtaining range data at each "point" in the scene) or by using a large laser beam that illuminates the entire scene in one flash and then 
measuring the reflected signal with a detector array that obtains numerous range points simultaneously.

The visible spectrum is part of the electromagnetic spectrum that is detectable to the human eye. The range of the wavelengths of visible light to a typical human eye is about 390 to $700 \mathrm{~nm}$ [4]. Human eye response can extend out to $750 \mathrm{~nm}$ wavelength for some individuals. It is undesirable to have LIDAR with visible lights installed on vehicles due to the potential for distraction as well as eye safety concerns associated with lasers. As a result, most automotive LIDAR systems use lasers that operate in the near-infrared portion of the electromagnetic spectrum. A search of the automotive LIDAR shows that most sensors operate in the wavelength range 785-1064 $\mathrm{nm}$ [5-9]. We have not found any specific automotive LIDAR examples operating under $785 \mathrm{~nm}$. The data in Section 3 shows that IR reflectivity of common clothing material is high in the near-infrared wavelength range of 800-1200 nm.

\section{B. LiDAR operation principle}

Obtaining IR reflectivity measurements accurately requires accounting for numerous factors, including the characteristics of the intervening atmosphere as well as the geometry between the laser source, objects being interrogated, and the optical receiver. The basic laser range equation for a simplified LiDAR system is shown in equation (1), where $P_{R E C}$ represents the received power and $P_{T}$ represents transmitted power, $\eta$ represents the receiver quantum efficiency, $R$ is the range to the target, $A_{R E C}$ is the area of the receiver, $A_{\text {TARGET }}$ is the area of the target, $D i v_{\text {TRANS }}$ is the laser beam divergence before reflection, $D i v_{R E F}$ is the laser beam divergence after reflection, and $\rho$ represents the reflectance of the target. It should be noted that equation 1 neglects the effects of the intervening atmosphere between the LIDAR system and the targets in the scene and only captures the effects of the sensor and target behavior.

$$
P_{R E C}=P_{T} \cdot \frac{\eta}{\pi^{2} R^{4}} \cdot \frac{A_{R E C}}{\operatorname{Div}_{T R A N S}} \cdot \frac{\rho A_{\text {Target }}}{\text { Div }_{R E F}}
$$

The reflected laser pulse can be detected if the received power as determined in equation (1) provides an adequate signal to noise ratio (SNR) when compared to the noise level of the receiver. The range to the target is not obtained from equation (1) but is determined by multiplying the time of flight required for the laser pulse to travel from its source to a target and for the reflected pulse to travel back to the receiver (usually co-located with the source) by the speed of light and then dividing by two (due to the round-trip travel). This relationship assumes that the time of flight has been measured accurately by the LIDAR system.

A quick analysis of the laser range equation indicates that the received power level is determined principally by the target reflectance properties, assuming that the LIDAR system parameters are fixed and range is treated as the independent variable. Therefore, it is better for the LIDAR to operate in a wavelength range where the targets of interest are more reflective.
The appropriate specification of target infrared reflectance is essential to ensuring proper performance testing of LIDAR sensors. Incident light on a particular medium is affected by a number of different phenomena, including refraction, reflection, absorption, diffraction, and diffusion, while it propagates through the medium. The net effect on the behavior of the light varies widely depending on the properties of that particular medium; however some portion of the light will be reemitted with a spectrum of reflectance that is unique to that medium. Reflectance is defined here as the ratio of the reflected flux to the incident flux at a given wavelength and for a given measurement geometry. For a laser beam incident on a target such as a pedestrian mannequin, the reflected signal will contain some percentage of the incident power and may also have a different divergence based on the geometry of the target. Reflectivity is a directional property, so if a beam of incident radiation is reflected strongly into a single direction (such as with a mirror), the reflection is called specular and the surface is described as glossy. When the radiation is reflected into all directions with a uniform distribution, the reflection is called diffuse and the surface is describes as lusterless. Illustrations of these cases are shown in the Figure 1(a). Most real surfaces fall somewhere between these two extremes.

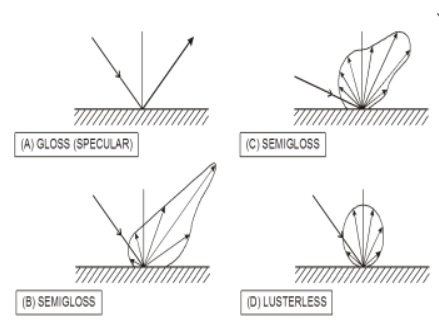

(a)

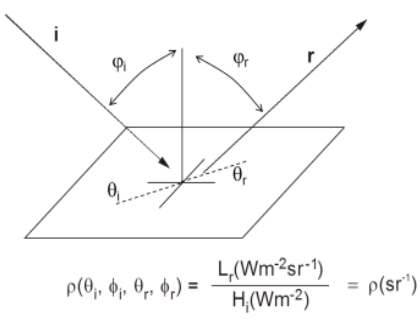

(b)
Figure 1. (a) Directional reflectivity examples and (b) bidirectional reflectance distribution function geometry.

It is important to note that reflectance is a bi-directional property that is dependent on the source, material, and observer geometry and can only be fully characterized by the Bi-directional Reflectance Distribution Function (BRDF) that describes reflectance for all combinations of source and observer angles as shown in Figure 1(b). These reflections are comprised of both a diffuse component, where reflections are scattered in all directions, and a specular component, where the light is reflected in accordance with Fresnel's law. If the BRDF is integrated over all angles, one can obtain the Total Hemispherical Reflectance (THR) for a sample. A surface that displays ideal diffuse reflectance where light is scattered equally in all directions is described as Lambertian. Diffuse reflectance is of particular interest for understanding the appearance of materials, since most naturally occurring surfaces, including fabrics, are diffuse reflectors. Assuming that clothing has diffuse reflectance properties significantly simplifies the testing process (by avoiding the need to test at numerous angles of incidence), but care must be taken to ensure that this assumption is valid in practice. This can be 
accomplished by measuring the reflectance at several different angles of incidence and reflection for a particular material to confirm that the variation in reflectance as a function of angle is minimal. More samples are better, but at least nine combinations of incidence and reflectance angles should be measured. This allows the measurement of three different reflection angles for each of three angles of incidence. For a Lambertian reflector, these measurements should indicate that the reflectance at any combination of incidence and reflection angle does not vary by more than $+/-20 \%$ from the value measured at the normal angle of incidence and reflection. These measurements should be performed on a representative sample of the material prior to using it in tests where Lambertian behavior is assumed.

\section{INFRARED REFLECTIVITY FOR A PEDESTRIAN MANNEQUIN}

Since the penetration depth of infrared light is on the order of micrometers, the IR reflectivity of pedestrians is determined by their skin and clothing. Here, we determine the IR reflectance of skin and clothing and specify the IR reflectance property for standard pedestrian mannequins.

\section{A. IR reflectance of pedestrian mannequin skin}

Human skin reflectivity in various spectrums has been measured numerous times $[10,11]$. Jacquez showed that in the $800 \mathrm{~nm}-1100 \mathrm{~nm}$ wavelength range, the IR reflectivity of white race skin is in the range from 0.44 to 0.60 . Cooksey presented the similar results for white skin and also provided that for black race skin is in the range from 0.40.55 , and that of Asian race skin is from 0.43 to 0.58 . The results were consistent in different experiments. Therefore, we recommend that the IR reflectance of the surrogate pedestrian mannequin skin to be in the range between 0.4 and 0.6 .

\section{$B$. IR reflectance of clothing fabric}

As will be shown later in the paper, the IR reflectivity of clothing is a function of wavelength, fabric material, fabric color, and fabric weaving method. According to $[12,13]$, natural material cotton and manmade material polyester are the most common clothing material (especially for male clothing). Based on the clothing color analysis for pedestrians in TASI 110 car naturalistic study [14], the representative color for adults upper clothing is black ( $\mathrm{RGB}=43,41,44)$, for adults lower clothing is deep dark blue $(\mathrm{RGB}=43,44,53)$, for children upper clothing is black ( $\mathrm{RGB}=48,46,48)$, and for children lower clothing is medium blue $(\mathrm{RGB}=65,71,88)$. So the next problem is to determine the corresponding IR reflectivity for the common cloth fabric material in these color ranges.

When specifying the infrared reflectance for clothing used on the pedestrian mannequin, it is necessary to consider that the dyes used to give fabrics color are formulated for the visible portion of the spectrum (400-700 $\mathrm{nm}$ ) and may have different properties at longer wavelengths where typical LIDAR systems operate. In addition, fabrics are actually comprised of layers of fibers, and depending on the thickness of the fabric and the density of the fibers, there may be some amount of reflection from the substrate underneath the fabric (e.g. skin or a different fabric) and therefore the apparent reflectance would represent the contributions from multiple layers. We measured 98 samples of cotton and polyester fabric in a wide variety of color with a variety of patterns from two vendors who supply fabric to the clothing industry [15]. These measurements were made using a Cary 5E spectrophotometer with the diffuse reflectance accessory installed over a wavelength range of 400 to $2000 \mathrm{~nm}$ with an interval of $1 \mathrm{~nm}$. The instrument was first baselined by measuring the reflectance of a reference sample comprised of a Lambertian Spectralon coating with near $100 \%$ reflectance across the whole spectral region. This was followed by measurement of a zero baseline where the sample holder was empty. The test article was then installed in the sample holder and its spectrum was recorded by the spectrophotometer and calibrated based on the the reference sample and zero baseline measurements. The full procedure is described in reference [15].

The description of the fiber samples are listed in Table 1. Figures 3 and 4 plot the percentage reflectance versus wavelength for a variety of samples of cotton and polyester fabric. It is readily apparent from this data that the reflectance values can change dramatically at wavelengths above $700 \mathrm{~nm}$ and are not necessarily consistent at longer wavelengths either. The reflectance changes significantly between wavelengths $700-800 \mathrm{~nm}$ for most fabrics. The reflectivity of most fabric are relatively stable in the wavelength range $800-1200 \mathrm{~nm}$. It is also apparent that reflectance values can vary significantly from sample to sample, even at a particular wavelength.

The median and standard deviation of the measured reflectance values of all samples at $50 \mathrm{~nm}$ intervals in 700$1100 \mathrm{~nm}$ (in which most automotive LiDAR operate) are shown in Table 2. The samples displayed a one-sigma range of reflectance values from approximately $41 \%$ to $75 \%$ in wavelength range $800-1100 \mathrm{~nm}$.

TABLE I. DESCRIPTION OF FABRIC SAMPLES USED FOR IR REFLECTANCE MEASUREMENTS. MEASURED REFLECTANCE AT 750 NM AND BAND-AVERAGED REFLECTANCE AND STANDARD DEVIATION BETWEEN 800-1100 NM ARE SHOWN FOR EACH SAMPLE.

\begin{tabular}{|c|c|c|c|c|c|c|}
\hline Code & Name & Color & Type & Material & $750 \mathrm{~nm}$ & $800-1100 \mathrm{~nm}$ \\
\hline 20699 & Wailea Coast Sun Swatch & Ebony & Art & Cotton & 11 & $12 \pm 0.95$ \\
\hline 20771 & Orchid Cove Sun N Shade Swatch & Black & Art & Cotton & 3 & $4 \pm 0.36$ \\
\hline 25929 & African Print Swatch & Multi animal print & Art & Cotton & 37 & $55 \pm 1.37$ \\
\hline 26491 & African Print Swatch & Blue Orange Black Whi & Art & Cotton & 54 & $62 \pm 0.29$ \\
\hline 27401 & Western Style Swatch & Multi-colored on Red & Art & Cotton & 69 & $69 \pm 0.40$ \\
\hline
\end{tabular}




\begin{tabular}{|c|c|c|c|c|c|c|}
\hline 27735 & African Print Swatch & Burg, green blue blac & Art & Cotton & 48 & $53 \pm 0.18$ \\
\hline 27885 & Colorful Sneakers Swatch & Multi-colored on Turq & Art & Cotton & 48 & $63 \pm 0.36$ \\
\hline 27888 & Sassy Sealife Swatch & Blue and Orange on Gr & Art & Cotton & 51 & $62 \pm 0.44$ \\
\hline 27896 & Hawaiian Print Swatch & Yellow Orange Green R & Art & Cotton & 60 & $61 \pm 0.19$ \\
\hline 29852 & Rocket Ships Swatch & Multi Brights on Blac & Art & Cotton & 45 & $61 \pm 1.16$ \\
\hline 32787 & Dolphins Swatch & Shades of Blue & Art & Cotton & 51 & $63 \pm 0.55$ \\
\hline 34181 & African Animals Swatch & Multi & Art & Cotton & 49 & $57 \pm 1.93$ \\
\hline 34580 & Cotton Flannel Print Swatch & Multi on Blue & Art & Cotton & 48 & $55 \pm 2.31$ \\
\hline 34851 & Patriotic Print Swatch & Red/White/Blue/Black & Art & Cotton & 48 & $54 \pm 2.00$ \\
\hline 34869 & Hawaiian Print Swatch & Multi & Art & Cotton & 32 & $41 \pm 0.49$ \\
\hline 35634 & All Sports Swatch & Multi on Navy & Art & Cotton & 27 & $37 \pm 1.34$ \\
\hline 35979 & Color Quake Swatch & Multi on Shades of $\mathrm{Pu}$ & Art & Cotton & 38 & $42 \pm 0.40$ \\
\hline 35988 & Hawaiian Sunset Swatch & Multi & Art & Cotton & 46 & $55 \pm 2.77$ \\
\hline 35989 & American Heritage Swatch & Multi & Art & Cotton & 57 & $60 \pm 0.58$ \\
\hline 36267 & Lahaina Swatch & Black & Art & Cotton & 4 & $4 \pm 0.08$ \\
\hline 36476 & Hawaiian Print Swatch & Multi on Green & Art & Cotton & 52 & $57 \pm 0.32$ \\
\hline 36477 & Hawaiian Print Swatch & Multi on Red & Art & Cotton & 54 & $57 \pm 0.54$ \\
\hline 36495 & Ropin Swatch & Multi on Tan & Art & Cotton & 54 & $56 \pm 0.49$ \\
\hline 36832 & Surboard Collection Swatch & Multi on Pink & Art & Cotton & 55 & $55 \pm 0.44$ \\
\hline 26460 & African Print Swatch & Red, Green, Black,Yel & Patterned & Cotton & 57 & $59 \pm 0.31$ \\
\hline 26474 & African Print Swatch & Yellow Green Black Or & Patterned & Cotton & 32 & $46 \pm 1.48$ \\
\hline 26690 & Cotton Polka Dots Swatch & Black with White dots & Patterned & Cotton & 35 & $57 \pm 1.98$ \\
\hline 26696 & Cotton Polka Dots Swatch & Multi-colored on Blac & Patterned & Cotton & 57 & $57 \pm 0.37$ \\
\hline 26729 & Cotton Polka Dots Swatch & Royal Blue/White & Patterned & Cotton & 63 & $68 \pm 0.23$ \\
\hline 26754 & Cotton Polka Dots Swatch & Blue Red Yellow White & Patterned & Cotton & 58 & $59 \pm 0.41$ \\
\hline 31809 & Seersucker Shirting Swatch & Pink, Green, Yellow & Patterned & Cotton & 51 & $56 \pm 0.43$ \\
\hline 34668 & Camouflage Twill Swatch & Brown/Tan/Khaki & Patterned & Cotton & 25 & $47 \pm 11.18$ \\
\hline 34671 & Camouflage Print Swatch & Digital & Patterned & Cotton & 51 & $61 \pm 3.80$ \\
\hline 34856 & Patriotic Print Swatch & Red/White & Patterned & Cotton & 56 & $58 \pm 0.52$ \\
\hline 35197 & Seersucker Shirting Swatch & Turquoise/Navy Check & Patterned & Cotton & 33 & $54 \pm 1.96$ \\
\hline 35645 & Cotton Flannel Print Swatch & Multi & Patterned & Cotton & 61 & $69 \pm 0.68$ \\
\hline 35811 & African Print Swatch & Green/Red/Black/Purpl & Patterned & Cotton & 42 & $55 \pm 1.24$ \\
\hline 36364 & Seersucker Shirting Swatch & Pink/Purple Plaid & Patterned & Cotton & 59 & $59 \pm 0.53$ \\
\hline 37368 & Retro Dots Swatch & Blue/Lime/Ivory & Patterned & Cotton & 60 & $62 \pm 0.20$ \\
\hline 37665 & Camouflage Print Swatch & Blues & Patterned & Cotton & 15 & $19 \pm 0.56$ \\
\hline 29982 & Cotton T-Shirt Knit Swatch & Prism Violet & Solid & Cotton & 66 & $72 \pm 0.20$ \\
\hline 29995 & Cotton T-Shirt Knit Swatch & chintz rose & Solid & Cotton & 78 & $77 \pm 0.66$ \\
\hline 29999 & Cotton T-Shirt Knit Swatch & Blue Bell & Solid & Cotton & 69 & $69 \pm 0.25$ \\
\hline 30004 & Cotton T-Shirt Knit Swatch & Chili Pepper & Solid & Cotton & 67 & $70 \pm 0.61$ \\
\hline 30008 & Cotton T-Shirt Knit Swatch & Pastel Blue & Solid & Cotton & 71 & $71 \pm 0.26$ \\
\hline 30009 & Cotton T-Shirt Knit Swatch & Sunkist Coral & Solid & Cotton & 68 & $68 \pm 0.26$ \\
\hline 30013 & Cotton T-Shirt Knit Swatch & Pale Banana & Solid & Cotton & 68 & $68 \pm 0.24$ \\
\hline 31163 & Cotton T-Shirt Knit Swatch & Black & Solid & Cotton & 47 & $67 \pm 1.12$ \\
\hline 36411 & Cotton Interlock Swatch & Lime & Solid & Cotton & 62 & $66 \pm 0.66$ \\
\hline 36412 & Cotton Interlock Swatch & Stop Sign & Solid & Cotton & 68 & $69 \pm 0.20$ \\
\hline 36413 & Cotton Interlock Swatch & Royal Blue & Solid & Cotton & 42 & $59 \pm 8.45$ \\
\hline 20764 & Tangier Sun N Shade Swatch & Sage & Art & Polyester & 52 & $53 \pm 0.99$ \\
\hline 28276 & Seaside Lily Sun N Shade by Wa & Key Lime & Art & Polyester & 54 & $68 \pm 0.35$ \\
\hline 28277 & Isla Bonita Sun N Shade by Wav & Ocean & Art & Polyester & 65 & $70 \pm 0.42$ \\
\hline 28278 & Wailea Coast Sun N Shade by Wa & Flax & Art & Polyester & 70 & $72 \pm 0.65$ \\
\hline 28282 & Essence Sun N' Shade Waver1 & Onyx & Art & Polyester & 24 & $23 \pm 2.35$ \\
\hline 33785 & Sadie Swatch & Azalea & Art & Polyester & 54 & $57 \pm 0.54$ \\
\hline 33787 & Whim Swatch & Mist & Art & Polyester & 64 & $64 \pm 1.42$ \\
\hline 33788 & Curvature Swatch & Foliage & Art & Polyester & 25 & $30 \pm 2.19$ \\
\hline 33793 & Fiji Swatch & Midnight & Art & Polyester & 31 & $31 \pm 2.99$ \\
\hline 33796 & Jefferson Swatch & Café & Art & Polyester & 29 & $29 \pm 1.10$ \\
\hline 20766 & Biscayne Bay Sun N Shade Swatch & Dune & Patterned & Polyester & 41 & $46 \pm 0.84$ \\
\hline 20769 & Rodeo Drive Sun N Shade Swatch & Sea Spray & Patterned & Polyester & 29 & $31 \pm 1.04$ \\
\hline 20770 & Beach Umbrella Sun N Shade Swatch & Pink/Black & Patterned & Polyester & 67 & $67 \pm 0.69$ \\
\hline 20772 & Marina Del Ray Sun N Shade Swatch & Surf & Patterned & Polyester & 25 & $47 \pm 4.99$ \\
\hline 28279 & Beach Umbrella Sun N Shade by & Flax & Patterned & Polyester & 23 & $27 \pm 0.80$ \\
\hline 33794 & Bistro Stripe Swatch & Midnight & Patterned & Polyester & 45 & $46 \pm 1.15$ \\
\hline 33799 & Larrabee Swatch & Mocha & Patterned & Polyester & 18 & $19 \pm 0.81$ \\
\hline 36268 & La Jolla Stripe Swatch & Aqua & Patterned & Polyester & 51 & $54 \pm 0.57$ \\
\hline 28280 & Sunburst Sun N Shade by Waver1 & Chocolate & Solid & Polyester & 51 & $75 \pm 0.91$ \\
\hline 31039 & Polyester Shantique Swatch & Lilac & Solid & Polyester & 69 & $68 \pm 0.33$ \\
\hline 31498 & Polyester Charmeuse Swatch & Ocean & Solid & Polyester & 59 & $58 \pm 0.48$ \\
\hline 32750 & Polyester Charmeuse Swatch & Poppy & Solid & Polyester & 63 & $61 \pm 0.41$ \\
\hline 33512 & Polyester Charmeuse Swatch & Canteloupe & Solid & Polyester & 57 & $59 \pm 0.57$ \\
\hline
\end{tabular}




\begin{tabular}{|l|l|l|l|c|c|c|}
\hline 33907 & Polyester Poplin Swatch & White & Solid & Polyester & 72 & $72 \pm 0.25$ \\
\hline 33908 & Polyester Poplin Swatch & Ivory & Solid & Polyester & 56 & $55 \pm 0.38$ \\
\hline 33909 & Polyester Poplin Swatch & Gray & Solid & Polyester & 69 & $70 \pm 0.21$ \\
\hline 33910 & Polyester Poplin Swatch & Green & Solid & Polyester & 24 & $70 \pm 1.86$ \\
\hline 33911 & Polyester Poplin Swatch & Black & Solid & Polyester & 49 & $69 \pm 0.76$ \\
\hline 33912 & Polyester Poplin Swatch & Copen & Solid & Polyester & 69 & $70 \pm 0.25$ \\
\hline 33913 & Polyester Poplin Swatch & Burgundy & Solid & Polyester & 68 & $71 \pm 0.53$ \\
\hline 33914 & Polyester Poplin Swatch & Royal Blue & Solid & Polyester & 52 & $68 \pm 2.21$ \\
\hline 34661 & Polyester Poplin Swatch & Champagne & Solid & Polyester & 57 & $57 \pm 0.43$ \\
\hline 34662 & Polyester Poplin Swatch & Banana & Solid & Polyester & 56 & $56 \pm 0.51$ \\
\hline 34705 & Sunburst Sun N Shade Swatch & Keylime & Solid & Polyester & 51 & $74 \pm 3.33$ \\
\hline 35203 & Polyester Poplin Swatch & Light Blue/Navy Cross & Solid & Polyester & 34 & $58 \pm 3.31$ \\
\hline 35204 & Polyester Poplin Swatch & Tan/Brown Crossdye & Solid & Polyester & 33 & $58 \pm 3.81$ \\
\hline 36277 & FR Poplin Swatch & Chili & Solid & Polyester & 72 & $73 \pm 0.24$ \\
\hline 36282 & FR Poplin Swatch & Pistachio & Solid & Polyester & 74 & $74 \pm 0.23$ \\
\hline 36342 & Polyester Poplin Swatch & Navy & Solid & Polyester & 48 & $56 \pm 0.25$ \\
\hline 36343 & Polyester Poplin Swatch & Hunter & Solid & Polyester & 51 & $55 \pm 0.37$ \\
\hline 36344 & Polyester Poplin Swatch & Red & Solid & Polyester & 56 & $56 \pm 0.38$ \\
\hline 37140 & Polyester Charmeuse Swatch & Sunshine Yellow & Solid & Polyester & 49 & $51 \pm 0.32$ \\
\hline 37141 & Polyester Charmeuse Swatch & Cyan & Solid & Polyester & 39 & $61 \pm 0.61$ \\
\hline 37701 & Polyester Poplin Swatch & Mango & Solid & Polyester & 56 & $56 \pm 0.42$ \\
\hline
\end{tabular}

Cotton Spectral Reflectances

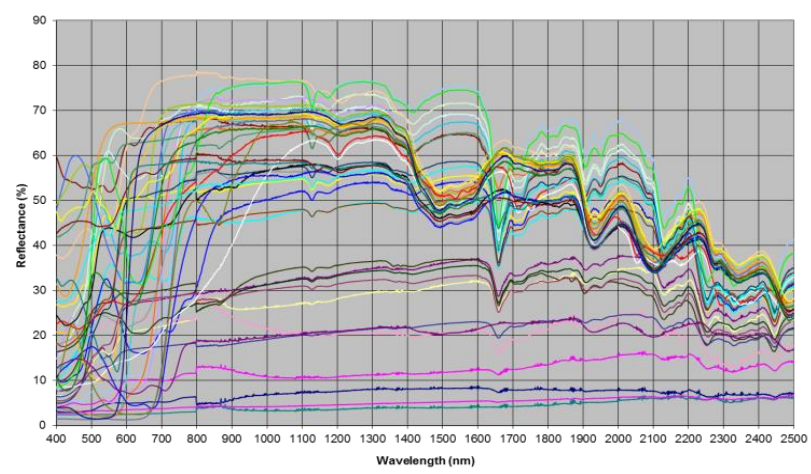

Figure 2. Measured spectral total hemispherical reflectance for cotton samples.

Polyester Sample Reflectances

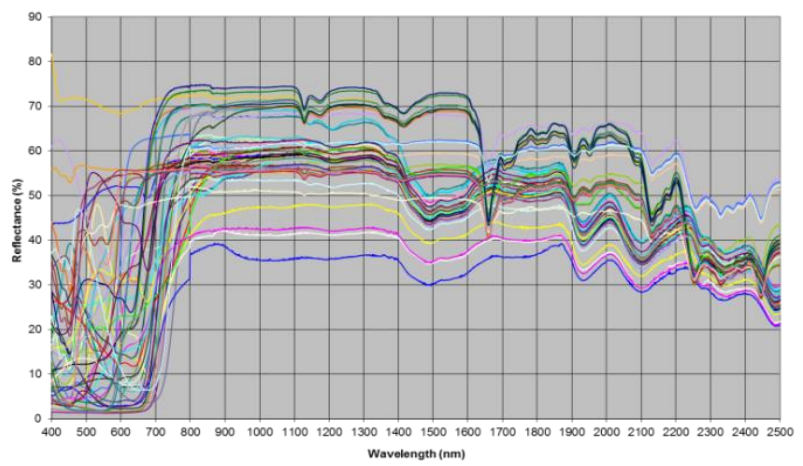

Figure 3. Measured spectral total hemispherical reflectance for polyester samples.

Therefore, we recommend the IR reflectance of the pedestrian mannequin clothing fabric be specified as the median $\pm(0.75$ Sigma $)$ based on the measured samples. In other words, we recommend that the IR reflectance is between $46-70 \%$ in the wavelength range from 800 to $1100 \mathrm{~nm}$ and between 40 and $64 \%$ in the range from 750 to $\underline{800 \mathrm{~nm}}$. In addition, the fabric shall be sufficiently thick such that it does not transmit any light through to the substrate of the mannequin that could result in a different apparent reflectance.

TABLE II. REFLECTANCE STATISTICS OF ALL MEASURED SAMPLES

\begin{tabular}{|r|c|c|c|}
\hline $\begin{array}{c}\text { Wavelength } \\
\text { (nm) }\end{array}$ & $\begin{array}{c}\text { Median } \\
\text { Reflectance (\%) }\end{array}$ & $\begin{array}{c}\text { Standard } \\
\text { Deviation (\%) }\end{array}$ & $\begin{array}{c}\text { One-Sigma } \\
\text { Range (\%) }\end{array}$ \\
\hline $\mathbf{7 0 0}$ & 42 & 20 & $22-62$ \\
\hline $\mathbf{7 5 0}$ & 52 & 17 & $35-69$ \\
\hline $\mathbf{8 0 0}$ & 57 & 16 & $41-73$ \\
\hline $\mathbf{8 5 0}$ & 58 & 16 & $42-74$ \\
\hline $\mathbf{9 0 0}$ & 58 & 16 & $42-74$ \\
\hline $\mathbf{9 5 0}$ & 58 & 16 & $42-74$ \\
\hline $\mathbf{1 0 0 0}$ & 59 & 16 & $43-75$ \\
\hline $\mathbf{1 0 5 0}$ & 59 & 16 & $43-75$ \\
\hline $\mathbf{1 1 0 0}$ & 59 & 16 & $43-75$ \\
\hline
\end{tabular}

\section{IR ReFlectance Measurement Methods}

The IR reflectance of the standard pedestrian mannequin should be measured to ensure it satisfies the specifications. The diffuse reflectance of the fabric selected shall be measured versus wavelength using a spectrophotometer (such as a Cary 5E) using the procedure described in Section III.B. These instruments typically use a broadband light source and diffraction gratings to produce monochromatic light that illuminates a sample. The reflected light is then collected using an integrating sphere (usually part of a diffuse reflectance accessory) which measures all of the light reflected from the sample regardless of the reflection angle. This measurement is then compared to the measurement for calibrated samples to determine an absolute reflectance value which describes the total hemispherical reflectance of the sample. 
In addition to measuring the diffuse reflectance, the BRDF of the sample at several discrete wavelengths should also be measured. These measurements shall not deviate by more than $20 \%$ from the value measured at the normal to the sample for a fabric that is a diffuse reflector. The BRDF is measured by illuminating a sample with a source placed at a particular incidence angle and then measuring the reflected light with a sensor placed at a known reflection angle. The wavelength of the illumination source is limited to a particular value through use of narrow band filters. This process is repeated for several different combinations of angles of incidence and reflection to fully characterize the BRDF. When samples are isotropic about their normals, it is not necessary to vary the incident azimuth. If the sample has surface structure that exhibits a preferred (as opposed to random) direction, the BRDF may vary significantly as a function of incidence angle; in this case BRDF measurements at several incident azimuth angles are required to fully characterize such a sample. The discrete values of a relative BRDF serve to characterize the reflectance of the sample. These relative BRDFs are then integrated and equated to the directional reflectance measured at the same incidence angle. This yields an absolute BRDF [16].

For situations where in situ measurements are required or preferred, infrared reflectance can also be measured using a portable spectrometer such as an Ocean Optics Jaz Spectrometer (http://oceanoptics.com/ product/jazspectrometer/) as described in the EAMA Articulated Pedestrian Target Specifications [2]. Before the start of the measurement, the device must be calibrated with a NISTtraceable reflection standard with a reflectance of $99 \%$. The calibration should then be verified using reflectance standards with reflectance of $25 \%$ and $50 \%$. The measurement shall be conducted with a special reflectance attachment which ensures a defined distance and angle between the probe and target. The measurement shall be performed at three separate points on the target with measurements taken at $45^{\circ}$ and $90^{\circ}$ for each point. The average of the three measurements at each angle will determine the reflectance for that material. The measured value at $45^{\circ}$ shall not differ by more than $20 \%$ from the value measured at $90^{\circ}$ to ensure diffuse behavior of the fabric.

\section{CONCLUSION}

The surrogate pedestrian mannequin is an essential tool for the performance evaluation of pedestrian autonomous emergency braking system. The IR reflectivity of the visible skin and clothing of the mannequin need to represent that of real pedestrians. This paper described a process and method to determine the IR reflectance of pedestrians and recommended the IR reflectance range for the standard surrogate pedestrian mannequin for AEB performance testing is between $46-70 \%$ in the wavelength range from 800 to $1100 \mathrm{~nm}$ and between 40 and $64 \%$ in the range from 750 to $800 \mathrm{~nm}$. These values will ensure that the pedestrian mannequin reflectance will be representative of actual pedestrians that might be encountered by an AEB system in real world situations. As AEB usage becomes more widespread, continuous data collection and monitoring of fabric samples should be performed to ensure that these standards continue to be representative of pedestrian reflectance as new materials are introduced and clothing trends evolve.

\section{REFERENCES}

[1] Qiang Yi, Stanley Chien, et. al, "Mannequin Development for Pedestrian Pre-Collision System evaluation," $17^{\text {th }}$ IEEE Int. conf. on Intelligent Trans. Sys., Oct 8-11, 2014, Qingdao, China.

[2] "Articulated Pedestrian Target Specifications Version 1.0", European Automobile Manufacturers Association, Oct 2015.

[3] Heath Albrecht, "Pedestrian Test Mannequins Objective Criteria for Evaluating Repeatability and Accuracy of PCAM Systems," http://www.nhtsa.gov/DOT/NHTSA/NVS/Public\%20Meetings/SAE/2 015/Pedestrian\%20Test $\% 20$ Mannequins\%20Objective\%20Criteria(fin al).pdf (May 2016).

[4] Cecie Starr (2005). Biology: Concepts and Applications. Thomson Brooks/Cole.ISBN 0-534-46226-X.

[5] Velodyne HDL-64E Data Sheet, http://velodyneLiDAR.com/ docs/datasheet/63-9194_Rev-D_HDL64E_Data\%20Sheet_Web. pdf , May 16, 2016.

[6] Eldada, Louay, "Today's LiDARs and GPUs Enable Ultra-accurate GPS-free Navigation with Affordable SLAM.” 2014 GPU Technology Conference, San Jose, CA on March 27, 2014.

[7] Comparison of laser range scanners (LIDARs), http://www.phoenixaerial.com/information/LiDAR-comparison (May 16, 2016).

[8] Trilumina LiDAR IR Laser Array Field, http://www.trilumina.com (May 16, 2016).

[9] Hokuyo Scanning Range Finder, https://www.hokuyoaut.jp/02sensor/07scanner/urg_04ln.html (May 16, 2016)

[10] John A. Jacquez, John Huss, Wayne McKeehan, James M. Dimitroff, Hans F. Kuppenheim, "Spectral Reflectance of Human Skin in the Region 0.7-2.6 $\mu$," Zournal of Applied Physiology Published 1 $\begin{array}{llllll}\text { November } & 1955 \quad \text { Vol. } & 8 & \text { no. } 3, & \text { 297-299, }\end{array}$ http://jap.physiology.org/content/8/3/297.full-text.pdf + html

[11] Catherine C. Cooksey, Benjamin K. Tsai, and David W. Allen, "A collection and statistical analysis of skin reflectance signatures for inherent variability over the $250 \mathrm{~nm}$ to $2500 \mathrm{~nm}$ spectral range,", Proc. of SPIE Vol. 9082, 908206 . 2014, http://www.nist.gov/customcf/get_pdf.cfm?pub_id=916057

[12] Cotton from field to Fabric, https://www.cotton.org/pubs/ cottoncounts/fieldtofabric/upload/Cotton-From-Field-to-Fabric-129kPDF.pdf (May 14, 2016)

[13] Cotton for Nonwovens: A Technical Guide. August 10, 2008]; Available from: $\mathrm{http}: / / \mathrm{www} . c 0 t t o n i n c . c o m /$ product/ NonWovens/ Nonwoven-Technical-Guide/

[14] Libo Dong, Stanley Chien, David Good, Kai Yang, Yaobin Chen, Rini Sherony, and Hiroyuki Takahashi , "Determination of Pedestrian Mannequin Clothing Color for Evaluation of Pedestrian Pre-Collision Systems (PCS)," The 24th International Technical Conference on the Enhanced Safety of Vehicles (ESV2015), Gothenburg, Sweden on June 8-11, 2015.

[15] Haran, Terence, "Short-Wave Infrared Diffuse Reflectance of Textile Materials." Thesis, Georgia State University, 2008.

[16] B. P. Sandford, "Infrared Reflectance Properties of Aircraft Paints", AFGL-TR-84-0307, USAF Geophysics Laboratory, Hanscomb AFB, 1984. 